cardiovascular disease (30/1000/yr), 1044 from cancer (17/1000/yr]); 709 from respiratory (12/1000/yr); and 782 (13/1000/yr) from other causes. Overall mortality rates were higher among current-smokers compared with never-smokers (HR 1.49, 95\% CI 1.34 to 1.65), due to increased mortality from cardiovascular disease $(1.35,1.15$ to 1.58$)$, cancer (1.71, 1.39 to 2.09), and respiratory disease (2.27, 1.75 to 2.95$)$. Among smokers, mortality rates were linearly related to the number of cigarettes smoked. Former-smokers had only moderately higher overall mortality rates compared with never-smokers (HR 1.16, 1.07 to 1.25$)$, chiefly due to continued increased risks of death from cancer (1.21, 1.03 to 1.42$)$ and respiratory disease (1.60, 1.29 to 1.98$)$.

Conclusions Among men who survived into their 70 s, continuing to smoke is associated with persistent excess vascular and non-vascular mortality.

\section{P2-27 DOES SOCIAL DISADVANTAGE IN EARLIER CHILDHOOD PREDISPOSE TO ONSET OF LIMITING LONGTERM ILLNESS (LLTI)/DISABILITY IN LATER CHILDHOOD? A POPULATION BASED STUDY USING THE UK ONS LONGITUDINAL STUDY (ONSLS)}

doi:10.1136/jech.2011.142976h.63

C Blackburn, ${ }^{*}$ N Spencer, J Read. University of Warwick, Coventry, UK

Introduction There is a lack of empirical evidence on the predictors and temporal ordering of factors associated with child disability.

Objective To examine the relationship between social disadvantage in earlier childhood and the onset of LLTI/D in later childhood in the UK ONSLS.

Methods Children born between the 1981 and 1991 UK censuses who became ONSLS members and were present at the 2001 census were identified. Data were extracted on those children who didn't have LLTI/D in 1991 but did in 2001 (index) and those with no LLTI/D on either occasion (comparison). A social disadvantage index (SDI) was constructed for 1991. Logistic regression models were fitted on LLTI/D with the SDI as the independent variable of interest adjusted for age, gender, lone parenthood, and ethnicity.

Results $60000+$ children became ONSLS members between 1981 and 1991 and were present at the 1991 and 2001 censuses. 52438 in the index and comparison groups had complete data. In bivariate analysis, social disadvantage, age, gender and lone parenthood but not ethnicity were significantly associated with the onset of LLTI/D in the index group. After adjustment for confounding, the SDI showed a finely graded association with onset of LLTI/D in the index group (most disadvantaged $\mathrm{OR}=2.12$ (1.77, 2.54); disadvantaged in two domains $\mathrm{OR}=1.45(1.20,1.75)$; disadvantaged in one domain $\mathrm{OR}=1.14(0.94,1.40))$.

Conclusions Social disadvantage in earlier childhood as a predisposing factor for the development of LLTI/D in later childhood and adolescence. Social disadvantage may be both cause and consequence of childhood LLTI/D.

\section{P2-28 FROM PROTOCOL TO PROGRESS: ESTABLISHING A REGISTRY OF CHILDREN AND YOUNG PEOPLE WITH DIABETES IN NORTH EAST ENGLAND AND NORTH CUMBRIA}

doi:10.1136/jech.2011.142976h.64

${ }^{1} \mathrm{~K}$ Blakey, ${ }^{*}{ }^{2} \mathrm{G}$ Johnson, ${ }^{1} \mathrm{R}$ McNally, ${ }^{1} \mathrm{~S}$ Court, ${ }^{3} \mathrm{~T}$ Cheetham. ${ }^{1}$ Institute of Health \& Society, Newcastle University, Newcastle Upon Tyne, UK; ${ }^{2}$ NHS Diabetes, Newcastle Upon Tyne, UK; ${ }^{3}$ Institute of Human Genetics, Newcastle University, Newcastle Upon Tyne, UK

Introduction The changing nature of diabetes in the young has underlined the importance of reliable data on where and how patients with diabetes present and live. In the former northern health region of England, an area encompassing North East England and North Cumbria, intriguing patterns in incidence and relationships with socio-economic status have been identified. The study aimed to establish a diabetes registry for children and young people in the former northern health region of England.

Methods NHS Diabetes provided regional paediatric networks with financial support to enable delivery of national outcomes and improve diabetes care in the young. During regional meetings, the North East Clinical Paediatric Network discussed a proposal to fund establishment of a regional registry for patients with diabetes aged $<18$ years. The plan was universally supported. Funding was used to employ a registry coordinator. All regulatory approval applications were completed within a rigorous and transparent ethical framework. User group involvement formed a cornerstone of the process; their feedback underpinned the production of all patient, parents and care givers documentation. A robust data security and protection policy was developed.

Results There has been significant learning regarding resources required to establish a diabetes registry including identification of sustainability issues. Methodologies have been documented and an algorithm has been generated. Data collection commenced in January 2011.

Conclusions Establishing a registry is a complex process requiring many legal and ethical considerations. The algorithm can be used as a generic template to initiate similar registries in other geographical areas or for other diseases types.

\section{P2-29 ASSOCIATION BETWEEN SELF-REPORTED DEPRESSION AND CHRONIC DISEASES: FINDINGS FROM A POPULATION BASED STUDY}

doi:10.1136/jech.2011.142976h.65

A Boing, ${ }^{*}$ G Mello, A Boing, R Moretti-Pires, K Peres, M Peres. Federal University of Santa Catarina (UFSC), Florianópilis, Santa Catarina, Brazil

Introduction Cross-sectional studies have reported associations between depression and chronic diseases, however it is necessary adjust the analysis for potential confounders. The aim of this study was to examine the association between depression and chronic diseases among adults after controlling for potential confounders.

Methods This is a population-based cross-sectional study with a sample of 1720 adults aged 20-59 years from Florianópolis, Brazil. Interviews and anthropometric measures were performed at adults' households in 2009 and included data about socio demographic characteristics, 11 self-reported chronic diseases, use of health services and physical activity at leisure time. Poisson regression models were carried out to estimate Prevalence Ratios (PR) for the association between depression and chronic diseases (none; one; two or more chronic diseases) after controlling for sex, age, marital status, income, physical activity at leisure time and hospitalisation in the last 12 months.

Results The prevalence of depression was $17.1 \%$ (95\% CI $14.9 \%$ to $19.2 \%$ ). Even after adjusting for potential confounders the association between depression and chronic diseases remained statistically significant. Those with one chronic disease presented a prevalence of depression 40\% (PR 1.4; 95\% CI 1.05 to 1.87) higher than people without disease; the prevalence of depression was $120 \%$ (PR 2.2; $95 \%$ CI 1.70 to 2.87) higher among those with two or more chronic diseases. Conclusion There is an association between depression and chronic diseases regardless potential confounders.

\section{P2-30 CHRONIC ILLNESS AND SUBJECTIVE WELL-BEING OF FAMILY MEMBERS}

doi:10.1136/jech.2011.142976h.66

C Booker, ${ }^{*}$ A Sacker. University of Essex, Colchester, Essex, UK

Background The hedonic definition of subjective well-being includes subjective perceptions of moods such as happiness and cognitive 